\title{
Research Paper: The Relationship Between Ruminating the Catastrophic Consequences of Bodily Changes and Positive Reappraisal and Practical Problem-Solving Strategies in Individuals With Illness Anxiety Disorder
}

\author{
Mina Elhamias ${ }^{* *}$ (D), Mohsen Dehghani ${ }^{1}$, Mahmood Heidari ${ }^{1}$, Ali Khatibi² ${ }^{\mathbb{D}}$ \\ 1. Department of Psychology, Faculty of Education and Psychology, Shahid Beheshti University, Tehran, Iran \\ 2. Interdisciplinary Program in Neuroscience, Bilkent University, Ankara, Turkey.
}

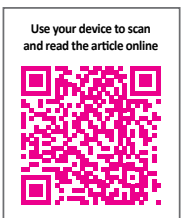

Cftation: Elhamiasl, M., Dehghani, M., Heidari, M., \& Khatibi, A. (2020). The Relationship Between Ruminating the Catastrophic Consequences of Bodily Changes and Positive Reappraisal and Practical Problem-Solving Strategies in Individuals With Illness Anxiety Disorder. Basic and Clinical Neuroscience, 11(5), 639-648. http://dx.doi.org/10.32598/bcn.9.10.240

oil http://dx.doi.org/10.32598/bcn.9.10.240

\section{Article info:}

Received: 12 May 2019

First Revision: 22 May 2019

Accepted: 05 Nov 2019

Available Online: 01 Sep 2020

Keywords:

Illness anxiety disorder, Emotion regulation, Interpretation bias, Catastrophizing, Bodily symptoms

\begin{abstract}
A B S T RA C T
Introduction: Cognitive emotion regulation is suggested to contribute to Illness Anxiety Disorder (IAD). Reappraisal and suppression are essential ER strategies with controversial data about their roles in IAD. Relevant studies are mostly limited to exploring these two strategies in individuals without such disorder. Therefore, we aimed to study the role of emotion regulation in the psychopathology of IAD by evaluating other ER strategies in illnessanxious individuals. Furthermore, we investigated the relationship between IAD and emotion regulation by targeting the role of interpretation bias for health-related information.

Methods: The study participants were 60 university students. They underwent a semistructured clinical interview to assess the presence or absence of IAD symptoms $(n=30 /$ group). They completed a battery of questionnaires measuring IAD, emotion regulation, and interpretation bias.

Results: The illness-anxious group applied significantly less reappraisal and refocus on planning and more rumination, catastrophizing, and acceptance strategies, compared to the controls. Besides, interpretation bias was positively correlated with rumination and catastrophizing; while its association with reappraisal and planning was negative.

Conclusion: Both functional (e.g. reappraisal \& planning) and dysfunctional strategies (e.g. rumination \& catastrophizing) contributed to the psychopathology of IAD. The biased interpretation of bodily information could make individuals prone to ruminate about the catastrophic consequences of bodily changes; such conditions interrupt fostering more positive reappraisal or practical problem-solving strategies.
\end{abstract}




\section{Highlights}

- The illness anxiety disorder group used different emotion regulation strategies, compared to the controls.

- The illness anxiety disorder group used less reappraisal and planning than the controls.

- The illness anxiety disorder group applied more dysfunctional emotion regulation strategies, compared to the controls.

- Interpretation bias was positively correlated with rumination and catastrophizing.

- Interpretation bias was negatively associated with reappraisal and planning.

\section{Plain Language Summary}

Worries about our health could help to detect a possible physical problem and to treat it before getting serious. However, severe preoccupation with the fear of severe illnesses can lead to excessive anxiety levels, known as illness anxiety disorder, that can interrupt daily life. Therefore, it is essential to understand which factors might make people prone to unnecessary worrying about bodily sensations. Impaired emotion regulation abilities, which refers to the strategies involved in down-regulating negative emotions, might be one of these factors. We studied if people with illness anxiety disorder might use different emotion regulation strategies, compared to those without illness anxiety. Besides, interpretation bias for health-related information (i.e. expecting the worse outcome for a health-threatening situation) is the main feature of illness anxiety. Therefore, we tested if there is a relationship between interpretation bias and emotion regulation in people with and without the illness anxiety disorder. Our results revealed that people with illness anxiety disorder used fewer appropriate emotion regulation strategies, such as reappraisal and planning, compared to those without such disorder. Illnessanxious individuals also showed higher rumination and catastrophizing, compared to the healthy ones. Our results also suggested that increases in interpretation bias were associated with enhanced rumination and catastrophizing and decreased functional reappraisal and planning. Therefore, individuals' inability to find alternative positive outcomes for a situation might make them think constantly about that situation. Passive catastrophic thinking, in addition, prevents people from engaging in more active solutions, such as planning to solve their health problems with more appropriate solutions. These results can be used to educate illness-anxious people to stop rumination about catastrophic interpretations and replace them with safer appraisals and plans to reduce their anxiety.

\section{Introduction}

I

llness Anxiety Disorder (IAD) refers to preoccupation with the fear of a serious illness that increases anxiety level and makes individuals vigilant to their health state (American Psychiatry Association, 2013). IAD can be considered as an independent disorder; however, it is a prevalent symptom of other anxiety disorders, such as Generalized Anxiety Disorder (GAD), specific phobia, and Obsessive-Compulsive Disorder (OCD) (See Abramowitz, Brigidi \& Foa, 1999; Deacon \& Abramowita, 2008). The prevalence of IAD and its associated frequent medical visits, as a dramatic burden on the healthcare system, demand a better understanding of the problem (Barsky, Ettner, Horsky \& Bates, 2001; Gorgen, Hiller \& Witthoft, 2014).
Emotion Regulation (ER) is a model that might increase our comprehension of IAD. Emotion dysregulation has been reported among the most essential psychological construct observable in approximately $75 \%$ of psychiatric disorders; it leads to experiencing negative emotions severely and uncontrollably, as they lack the required skills for managing and regulating their severe emotions (Campbell-Sills, Ellard \& Barlow, 2013). Gross and John (2003) suggests that cognitive reappraisal and expressive suppression are two major ER strategies. Cognitive reappraisal refers to the reinterpretation of an emotional event to change the meaning of that event and related emotions. Expressive suppression is an ER strategy to hide, control, or modify emotions and emotional behaviors (Gross \& John, 2003; Gross \& Levenson, 1993). Reappraisal is an appropriate method leading to the reduction of negative emotions, such as anxiety and depression; however, suppression is a maladaptive one associated with increased negative emotions in clinical 
and non-clinical samples (Koster, Rassin, Crombex, \& Naring., 2003; Gross \& Levenson, 1993; Gorgen et al., 2014). Previous studies suggested that suppression and reappraisal might have respectively positive and negative relationships with worry about health and preoccupation with somatic sensations (Bardeen \& Fergus, 2014). However, others have claimed that IAD and reappraisal are not related; they proposed that psychopathology is not related to functional strategies, like reappraisal. Gorgen et al. (2014) reported that disease phobia and disease conviction were not correlated with reappraisal. Additionally, in Fergus and Valentiner's study (2010), reappraisal was only related to the perceptual aspect of hypochondria (as sensitivity to innocuous bodily sensations), rather than the affective, behavioral, or cognitive ones. These studies indicated that dysfunctional ER strategies, like suppression, were involved in IAD and its components, including body vigilance. However, another study reported that none of these strategies were related to IAD (Gerolimatos \& Edelstein, 2012).

Furthermore, the literature on the relationship between interpretation bias for health-related information suggested as the main component of IAD, and ER strategies could be considered as supplementary evidence supporting the role of ER pathology in the development and maintenance of IAD. However, some studies are based on data on hypochondria rather than IAD; it is a novel diagnostic term replacing hypochondria with some features in common (e.g. fear of illness). Interpretation bias refers to the attribution of catastrophic meaning to ambiguous and threatening information. Based on this definition, individuals with hypochondriasis as well as those with IAD, compared to healthy groups, may consider more negative consequences when appraising health-related situations. These negative thoughts are expressed in the form of most catastrophic appraisals; in turn, they increase the perception of bodily sensations leading to a more catastrophic appraisal of health-related issues. Such a positive relationship between negative appraisal and the biased interpretation of health-threatening data has been supported by several studies (Haenen, de Jong, Schmidt, Stevens \& Visser, 2000; Weck, Neng, Richtberg \& Stangier, 2012). These data suggested that positive reappraisal, as an ER mechanism that enables individuals in selecting safer interpretations, might be an impaired strategy in IAD.

Additionally, by considering the role of suppression, individuals with IAD avoid situations that might activate illness schemas. This can occur by distracting or suppressing biased interpretations and undesired emotions. Applying suppression could make individuals ex- perience unwanted bodily sensations, such as pain and catastrophic thoughts more chronically; it prevents them from coping with the underpinning emotion (Cioffi \& Holloway, 1993; Gilliam et al., 2010; Wegner, Schneider, Carter \& White, 1987). IAD refers to the chronic experience of worries about illness-related sensations and catastrophic interpretations of them; accordingly, suppression might be considered among the involved mechanisms in such chronicity. Therefore, concerning the relationship between interpretation bias and ER strategies, both strategies could be involved in the maintenance of IAD.

Considering the above-mentioned inconsistent research findings on the relationship between IAD and ER strategies of reappraisal and suppression, a potential cause of data discrepancy might be the mere focus on populations without IAD. Comparing ER strategies between individuals with higher and lower levels of illness anxiety could endorse if a difference in ER is a possible maintenance factor in IAD. another issue concerning studies on ER and IAD was only measuring reappraisal and suppression. However, some other ER strategies might prone individuals vulnerable to IAD. Finding these strategies might help us in the better conceptualization of the role of ER strategies in IAD.

Accordingly, the current study aimed to examine ER strategies in two IAD and control groups. We hypothesized that while reappraisal, as a functional strategy, would be used more by the control group, in comparison to the IAD group subjects, the IAD group would apply a more dysfunctional strategy of suppression, compared to the controls. In addition, individuals with IAD, compared to the controls, would use other functional strategies, such as acceptance and refocus on planning less; while would apply other dysfunctional strategies, such as rumination and catastrophizing more. Then, to explore the effect of ER in IAD, we evaluated the relationship between ER strategies and interpretation bias for health-related information. We assumed that higher interpretation bias would be related to less functional strategies and more dysfunctional ones.

\section{Materials and Methods}

The IAD group samples were 30 students from Shahid Beheshti University; they were selected through a research participation enouncement requesting individuals with IAD symptoms. In this announcement, some of the IAD symptoms, including experiencing worries about health, checking body status, avoidance from healthrelated information or searching for such data, and being 
sensitive to bodily changes, were listed. Volunteer students were supposed to inform the experimenter using email or text. To confirm the presence of IAD symptoms, one of the authors (M.E.; MA in Clinical Psychology; 5 years of supervised \& independent practice in anxiety disorders) assessed the IAD symptoms in volunteers by a semi-structured interview. A serious medical condition, receiving psychiatric medications, and the diagnosis of any other psychiatric disorders, such as major depressive disorder, obsessive-compulsive disorder, panic disorder, and psychosis in the last 5 years were the research exclusion criteria. Considering the inclusion and the exclusion criteria of the study, 30 participants (15 females) were selected from 47 volunteers. Other 17 participants were excluded due to not meeting the study inclusion criteria for IAD (4); medical diseases (3); a history of or current episodes of another psychiatric disorder (6); using psychiatric medications (2), and being under psychotherapy due to dysfunctional IAD (2). Overall, none of the selected participants experienced dysfunctional IAD that affected their daily living activities. They also had no dysfunctional comorbid psychiatric disorder, such as major depressive disorder, obsessive-compulsive disorder, or psychosis. Three of the research participants reported experiencing worries in other areas, including education or personal life. However, such experiences were not dysfunctional enough to visit a psychologist or receive a diagnosis of Generalized Anxiety Disorder (GAD). The volunteers who were excluded from the study due to experiencing psychiatric disorders were given some clarifying information on their observed problem; they were suggested to visit a psychologist at the psychotherapy and counseling center of Shahid Beheshti University.

The control group members included 30 students from Shahid Beheshti University. They were selected through an announcement requesting individuals without IAD symptoms. Volunteer students were supposed to inform the experimenter using email or text. Volunteers whose gender and age range matched to the IAD group were invited for an interview. To clarify the absence of IAD symptoms, a semi-structured interview, assessing illness anxiety symptoms, was conducted by a clinical psychologist. Encountering a serious medical condition, being under medical or psychiatric medications, and experiencing other psychiatric disorders were the exclusion criteria for this group. Thirty participants (15 females) among 37 volunteers were determined as eligible to be included in the control group. Eight volunteers were excluded due to experiencing medical diseases (2); a history or presence of another psychiatric disorder (2); using psychiatric medications (2), and a recent history of surgery (1). However, one of the thirty volunteers could not attend the measurement session due to an unexpected medical problem.

\subsection{The following tools were used in the present re- search}

The Structured Clinical Interview for DSM-5, Clinician Version (SCID-5-CV; First, Williams, Karg, \& Spitzer, 2015): SCID-5-CV is a semi-structured interview guide to make a diagnosis based on the Diagnostic and Statistical Manual of Mental Disorders, 5th Edition (DSM-5) criteria. The Persian version of this manual was administered by the author (M.E) who holds a certified degree in Clinical Psychology, to check the existence and absence of IAD symptoms, respectively in the test and control groups.

Short Health Anxiety Inventory (SHAI; Salkovskis, Rimes, \& Warwick, 2002): SHAI is an 18-item 4-point Likert-type questionnaire that evaluates IAD, independent from physical health status. These items measure various aspects of IAD, such as health worries, the awareness of bodily sensations and changes, and the negative consequents of illnesses. This inventory has been reported to have appropriate validity and reliability (Salkovskis et al., 2002). The Persian version of this questionnaire was used in the current study. The Internal consistency of the Persian version of this questionnaire in the current study, calculated by Cronbach's alpha coefficient, was equal to 0.93 .

Whiteley Index (WI): WI is a 14-item inventory, targeting IAD. It is scored dichotomously and continuously; we used the continuous version of scoring in the current study. This measure has a strong convergent correlation $(\mathrm{r}=0.80)$ with other scales of IAD (Pilowsky, 1967). The Persian version of this questionnaire was used in the current study. The Internal consistency of the Persian version of this scale in the current study, calculated by Cronbach's alpha coefficient, equaled 0.94.

Cognitions About Body and Health Questionnaire (CABAH): CABAH is a 31-item 5-point Likert-type questionnaire, assessing 5 subscales of catastrophizing interpretation of bodily complains, autonomic sensations, bodily weakness, the intolerance of bodily complains, and health habits (Rief, Hiller, \& Margraf, 1998). The internal consistency of this scale in clinical and healthy populations was reported as 0.90 and 0.80 , respectively. The Persian version of this questionnaire was used in the current study. The internal consistency of the Persian version of this inventory in the current study was 
calculated by Cronbach's alpha coefficient as 0.93 . In the current study, we only used the catastrophizing interpretation of bodily complaints subscale as the index of interpretation bias toward health-related information.

Emotion Regulation Questionnaire (ERQ): ERQ is a 10 -item scale designed to explore cognitive reappraisal and expressive suppression. The original internal consistency of the questionnaire was reported as appropriate (Gross \& John, 2003). The Persian version of this scale was used in the current study. The internal consistency of the Persian version of this questionnaire in the current study, as calculated by Cronbach's alpha coefficient, for reappraisal and suppression subscales was 0.84 and 0.82 , respectively.

Cognitive emotion regulation questionnaire (CERQ): CERQ is a 36-item questionnaire that measures cognitive ER strategies in response to a stressful life event. Its subscales demonstrated acceptable internal consistency (Cronbach's $\alpha>0.70$ ) (Garnefski, Kraaij \& Spinhoven, 2001). The Persian version of this inventory was used in the current study. The internal consistency of the Persian version of the tool's subscales was calculated by Cronbach's alpha coefficient to range between 0.74 and 0.86 .

The present study was approved by the Ethics Committee of the Psychology Department at Shahid Beheshti University (Code: 30514). All study participants have read and signed the informed consent form at the beginning of the first session (interview session). The consent form contained information about the study purposes (individual differences in some psychological factors), the measurement methods, the associated risks and benefits, and the rules for withdrawal from the study. For recruiting the study participants with IAD, volunteers were called by advertising in public places at the university. Upon the expression of interest, not meeting the exclusion criteria, as well as considering equal gender ratio, they were invited to an interview; during which, they were individually interviewed by a clinical psychologist to examine the presence of IAD, according to the semistructured clinical interview. Those volunteers who met the IAD symptoms were selected as the IAD group samples. The subjects were informed by the experimenter that the current study consisted of conducting some questionnaires, evaluating individual differences in some psychological factors. Then, the date for the second session was set for them to meet the experimenter at the test lab for further measurements. In the second session, they were requested to complete the questionnaires after reading the instructions. After completing the test battery, the research participants were debriefed and the session was terminated. Following completing the experiment in the IAD group, volunteers for the control group were called by advertising in the public places of the University. Upon the expression of interest, being matched to the age range and gender of the IAD group, and not meeting the exclusion criteria, they were invited to an interview; during which, they were individually interviewed by a clinical psychologist to examine the absence of IDA according to the semi-structured clinical interview. Those who did not meet the IAD symptoms were selected as the controls. The procedures for signing a consent form, interview, and collecting data were the same as those for the IAD group.

\section{Results}

Before conducting the major statistical analysis, the data belonging to 3 participants ( 2 in the IAD group) were removed due to the high number of missing data. The descriptive statistics on 56 research participants' data indicated an equal gender ratio in the whole sample (28 females). Gender distribution in the IAD and control groups was close to each other, as well (15 females in the IAD group \& 13 female controls). the Independent Samples t-test data revealed no significant difference between male (Mean $\pm \mathrm{SD}=19.82 \pm 10.44) ; \mathrm{t}_{54}=-0.21, \mathrm{P}=0.83$ ) and female $\left.\mathrm{s}(\mathrm{Mean} \pm \mathrm{SD}=20.42 \pm 10.98) ; \mathrm{t}_{54}=-0.21, \mathrm{P}=0.83\right)$ samples in health anxiety levels, assessed by HAI. Male (Mean $\pm \mathrm{SD}=22 \pm 10.97) ; \mathrm{t}_{54}=-0.23, \mathrm{P}=0.81$ ) and female $\left(\mathrm{Mean} \pm \mathrm{SD}=22.71 \pm 11.87\right.$ ); $\mathrm{t}_{54}=-0.23, \mathrm{P}=0.81$ ) difference in health anxiety level measured by WI score was insignificant as well. The study groups matched according to age as well (health-anxious group: $\mathrm{Mean} \pm \mathrm{SD}=23.17 \pm 2.38$; controls: Mean $\pm \mathrm{SD}=23.82 \pm 2.61$ ).

To examine our research hypothesis about the lower levels of functional and higher levels of dysfunctional ER strategies in the IAD group, compared with the controls, we performed a Multivariate Analysis of Variance (MANOVA) while the subscales of ERQ, i.e. reappraisal and suppression, as well as the subscales of CERQ, i.e. positive reappraisal, refocus on planning, catastrophizing, rumination, acceptance, positive focusing, putting into perspective, self-blame, and other-blame was considered as the dependent variables and group were the fixed one.

The equality of covariance and error variance, as two main prerequisites of MANOVA, were assessed using Box's M and Levene's tests. The Box's M test result was not significant $(\mathrm{P}=0.51)$; therefore, the MANOVA assumption of homogeneity of covariance was approved. The Levene's test data for each dependent variable were 
Table 1. The results of descriptive statistics tests and MANOVA for between-group differences in ER strategies ${ }^{1}(n=29)$

\begin{tabular}{|c|c|c|c|c|c|}
\hline \multirow{2}{*}{ Variabels } & \multicolumn{2}{|c|}{ Mean士SD } & \multirow{2}{*}{ SS } & \multirow{2}{*}{ MS } & \multirow{2}{*}{$\mathbf{F}$} \\
\hline & IAD & Control & & & \\
\hline Reappraisal & $23.28 \pm 4.95$ & $27.78 \pm 5.04$ & 283.50 & 283.50 & 11.53 \\
\hline Suppression & $13.64 \pm 5.37$ & $14.78 \pm 4.60$ & 18.28 & 18.28 & 0.73 \\
\hline Positive reappraisal & $12.71 \pm 3.14$ & $15.10 \pm 3.40$ & 80.16 & 80.16 & 7.45 \\
\hline Refocus on planning & $13.92 \pm 2.82$ & $15.75 \pm 2.84$ & 46.44 & 46.44 & 5.76 \\
\hline Catastrophizing & $12.53 \pm 2.80$ & $9.75 \pm 3.40$ & 108.64 & 108.64 & 11.14 \\
\hline Rumination & $15.14 \pm 2.66$ & $11.50 \pm 3.38$ & 185.78 & 185.78 & 20.04 \\
\hline Acceptance & $14.32 \pm 2.49$ & $13.03 \pm 1.97$ & 23.14 & 23.14 & 4.57 \\
\hline Positive focusing & $12.50 \pm 2.44$ & $13.39 \pm 2.93$ & 11.16 & 11.16 & 1.53 \\
\hline Put into perspective & $12.92 \pm 2.85$ & $12.10 \pm 2.71$ & 9.44 & 9.44 & 1.21 \\
\hline Self-blame & $13.82 \pm 2.93$ & $13.50 \pm 2.84$ & 1.44 & 1.44 & 0.17 \\
\hline Other-blame & $9.46 \pm 3.58$ & $9.78 \pm 2.89$ & 1.44 & 1.44 & 0.13 \\
\hline
\end{tabular}

SD: Standard Deviation, SS: Sum of Squares; MS: Mean of Squares;

NEURSCIENCE

${ }^{1}$ The results of descriptive statistics and MANOVA for reappraisal, suppression, positive reappraisal, refocus on planning, catastrophizing, rumination, acceptance, positive focusing, put into perspective, self-blame, and other-blame in IAD and control groups.

insignificant ( $\mathrm{P}>0.05)$; accordingly, the error variance of all the variables was equal across two research groups. MANOVA revealed a significant multivariate main effect for ER strategy [Pillai's trace $=0.505, \mathrm{~F}_{11,44}=4.07$, $\mathrm{P}=0.001, \eta \mathrm{p}^{2}=0.50$, observed power $\left.=0.99\right]$. According to the univariate test data, the IAD group, compared to the controls, reported significantly lower levels of reappraisal $\left[\mathrm{F}_{1,5}=11.35, \mathrm{P}=0.001, \eta \mathrm{p}^{2}=0.17\right]$, positive reappraisal $\left[\mathrm{F}_{1,54}=7.45, \mathrm{P}=0.009, \eta \mathrm{p}^{2}=0.12\right]$, and refocus on planning $\left[\mathrm{F}_{1,54}=5.76, \mathrm{P}=0.02, \eta \mathrm{p}^{2}=0.09\right]$, but higher levels of acceptance $\left[\mathrm{F}_{1,54}=4.57, \mathrm{P}=0.03, \eta \mathrm{p}^{2}=0.07\right]$, rumination $\left[\mathrm{F}_{1,54}=20.04, \mathrm{P}=0.001, \eta \mathrm{p}^{2}=0.27\right]$, and catastrophizing $\left[\mathrm{F}_{1,54}=11.14, \mathrm{P}=0.002, \eta \mathrm{p}^{2}=0.17\right]$. The between-group differences were not significant for the other ER strategies. The relevant results are presented in Table 1.

To explore the specific relationship between IAD and each ER strategy, we used Pearson correlation analysis to evaluate the correlation between two illness anxiety indexes (i.e. HAI \& WI) and ER strategies (assessed by ERQ \& CERQ) in the total sample $(\mathrm{N}=56)$. Consistent with the t-test findings, the levels of IAD in HAI were negatively correlated with applying reappraisal $(\mathrm{r}=-0.50$, $\mathrm{P}<0.001)$, positive reappraisal $(\mathrm{r}=-0.34, \mathrm{P}<0.01)$, and refocus on planning $(\mathrm{r}=-0.32, \mathrm{P}<0.05)$. Besides, the extent of IAD in WI was negatively correlated with reap- praisal $(\mathrm{r}=-0.50, \mathrm{P}<0.001)$, positive reappraisal $(\mathrm{r}=-0.42$, $\mathrm{P}<0.001)$, and refocus on planning $(\mathrm{r}=-0.42, \mathrm{P}<0.001)$. The levels of rumination $(\mathrm{r}=0.50, \mathrm{P}<0.001)$ and catastrophizing $(\mathrm{r}=0.38, \mathrm{P}<0.01)$ were positively associated with IAD scores in HAI. Scores in WI were also positively correlated with rumination $(\mathrm{r}=0.54, \mathrm{P}<0.001)$ and catastrophizing $(\mathrm{r}=0.44, \mathrm{P}<0.001)$. Table 2 lists these significant correlations.

Furthermore, we explored the relationship between ER and IAD by targeting interpretation bias. For this purpose, we used the Pearson correlation analysis to investigate the relationship between interpretation bias and ER strategies in the total sample. As hypothesized, interpretation bias was negatively correlated with reappraisal $(\mathrm{r}=-0.34, \mathrm{P}<0.01)$ and refocus on planning $(\mathrm{r}=-$ $0.30, \mathrm{P}<0.05$ ), and positively associated with rumination $(\mathrm{r}=0.44, \mathrm{P}<0.001)$ and catastrophizing $(\mathrm{r}=0.28, \mathrm{P}<0.05)$. However, no significant correlation was detected between interpretation bias and suppression. The significant correlations are reported in Table 3.

\section{Discussion}

In this study, we investigated whether ER can explain the differences between IAD and healthy control 
Table 2. The relationship between health anxiety and ER strategies ${ }^{1}$

\begin{tabular}{|c|c|c|c|c|c|}
\hline Variable & $\begin{array}{c}\text { ERQ } \\
\text { Reappraisal }\end{array}$ & $\begin{array}{c}\text { CERQ } \\
\text { Positive Reappraisal }\end{array}$ & $\begin{array}{c}\text { CERQ } \\
\text { Rumination }\end{array}$ & $\begin{array}{c}\text { CERQ } \\
\text { Catastrophizing }\end{array}$ & $\begin{array}{c}\text { CERQ } \\
\text { Refocus on Planning }\end{array}$ \\
\hline SHAI & $-0.50^{*}$ & $-0.34^{* *}$ & $0.50 *$ & $0.38^{* *}$ & $-0.32 * * *$ \\
\hline WI & $-0.50^{*}$ & $-0.42^{*}$ & $0.54 *$ & $0.44^{*}$ & $-0.42^{*}$ \\
\hline
\end{tabular}

${ }^{*} \mathrm{P}<0.05 ; * * \mathrm{P}<0.01 ; * * * \mathrm{P}<0.001$

NEUR:SCIENCE

SHAI: Short Health Anxiety Inventory; WI: Whiteley Index; ERQ: Emotion Regulation Questionnaire; CERQ: Cognitive Emotion Regulation Questionnaire;

${ }^{1}$ Correlations between health anxiety indices (Short Health Anxiety Inventory and Whiteley Index) and the subscales of ER strategies.

Table 3. The relationship between interpretation bias and ER strategies

\begin{tabular}{ccccc}
\hline Variable & $\begin{array}{c}\text { ERQ } \\
\text { Reappraisal }\end{array}$ & $\begin{array}{c}\text { CERQ } \\
\text { Rumination }\end{array}$ & $\begin{array}{c}\text { CERQ } \\
\text { Catastrophizing }\end{array}$ & $\begin{array}{c}\text { CERQ } \\
\text { Refocus on planning }\end{array}$ \\
\hline $\begin{array}{c}\text { CABAH } \\
\text { Interpretation bias }\end{array}$ & $-0.34^{*}$ & $0.44^{* * *}$ & $0.28^{* *}$ & $-0.30^{* *}$ \\
\hline$* * * \mathrm{P}<0.001 ; * * \mathrm{P}<0.05, * \mathrm{P}<0.01 ;$ & & & & NEUR:\%SCIENCE \\
\hline
\end{tabular}

ERQ: Emotion Regulation Questionnaire; CERQ: Cognitive Emotion Regulation Questionnaire; CABAH: Cognitions About Body And Health.

groups. We observed that individuals with IAD used less reappraisal, compared to the controls; however, there was no such difference in suppression. The IAD group used more acceptance, rumination, and catastrophizing strategies and less positive reappraisal and refocus on planning, compared to the controls. Greater functional strategies of reappraisal and refocus on planning were associated with less interpretation bias; while the dysfunctional strategies of catastrophizing and rumination were positively correlated with interpretation bias.

The observed difference between the IAD and control samples in employing reappraisal suggested that reappraisal could be related to the IAD pathology. Bardeen and Fergus (2014) argued that less application of reappraisal was accompanied by higher levels of health-related worries and preoccupation with bodily sensations. However, the groups were not different based on adopting suppression. There is considerable inconsistency in the literature about the influence of suppression in IAD. Some studies supported the relationship between suppression and IAD and its components, including bodily vigilance (Gorgen et ak., 2014; Fergus, \& Valentiner, 2010); however, some others did not support these findings (Gerolimatos, \& Edelstein, 2012). Suppression is a dysfunctional strategy in the Gross model; however, some studies revealed that the functionality or dysfunctionality of reappraisal and suppression depends on the context (Bardeen, \& Fergus, 2014). Therefore, both study group members might have used suppression, but its consequences depend on the context.

We also explored whether other ER strategies, addressed as involved in IAD, may contribute to the differences between individuals with and without IAD. We found that refocus on planning was higher applied by the control group, compared to the IAD one. Refocus on planning reflects an active coping strategy that makes individuals take practical measures to cope with the problem rather than ruminating about it (Garnefski et al., 2001). To our knowledge, no study has investigated this strategy in IAD; however, some researchers reported that higher refocus on planning was associated with encountering less negative emotions, such as depression and anxiety (Kulpa, Zietalewicz, Kosowics, StypulaCiuba \& Ziolkowska, 2016). Furthermore, the effect of reduced refocus on planning in IAD could be explained better by rumination strategy. In this study, rumination was significantly higher in individuals with IAD, in comparison to the controls. Rumination refers to a maladaptive problem-solving style that forces individuals to constantly think about negative emotions and their possible causes, instead of engaging in more practical solutions (Garnefski et al., 2001). Therefore, a greater focusing on repetitive dysfunctional thoughts (i.e. rumination) could be accompanied by less focusing on active planning. Supporting this inference, it was reported that dysphoric students with higher scores in rumination could implement their plans with less possibility, compared to those 
with less rumination (Lyubomirsky, Tucker, Caldwell \& Berg, 1999). This inference was supported when we detected a significant negative correlation between the refocus on planning and rumination. Catastrophizing was another cognitive strategy, i.e. significantly higher among the studied individuals with IAD. This finding supports the data that individuals preoccupied with health issues used to consider more catastrophic outcomes for illnesses, compared with the control group (Weck et al., 2012). Moreover, acceptance, as a cognitive ER strategy, was significantly used further by the IAD group, compared to the controls. Acceptance refers to a strategy that enables an individual to accept what has occurred and to believe that it cannot be changed (Garnefski et al., 2001). Although acceptance, seems to be an adaptive process, its higher level can be an indicator of no control over the environment and an inability to influence events (Garnefski et al., 2001). Consistent with this finding, a study on ER and anxiety reported that further anxiety traits were positively correlated with more acceptance (Jacob \& Anto, 2016). Therefore, acceptance could sometimes refer to the negative form of regulating emotions and adapting to a situation (Garnefski et al., 2001).

The current study highlighted the effect of the ER strategies in IAD by investigating the relationship between ER and interpretation bias. Lower levels of reappraisal and planning along with higher levels of rumination and catastrophizing were correlated with higher interpretation bias for health-related data. This finding is in line with other studies on the impact of interpretation bias in IAD (Warwick \& Salkovskis, 1990; Marcus, Hughes \& Arnau, 2008). Constant thinking about catastrophic consequences of health-related information (i.e. rumination) might be related to individuals' inability to foster alternative appraisals for a situation and selecting the most positive and appropriate one (Akbari, Dehghani, Khatibi, \& Vervoort, 2016). Moreover, such ruminative catastrophic appraisals might be implicated by some individuals; they believe thinking about worse conditions might increase their ability in detecting a threat (e.g. bodily sensations) and coping with that (e.g. the treatment of a disease). The observed positive correlations between interpretation bias, catastrophizing, and rumination, as well as their negative association with reappraisal, could be considered as supporting evidence. This notion can also be endorsed by our findings concerning a negative association between interpretation bias and refocus on planning. Refocus on planning is a functional problemsolving strategy; it provides individuals an opportunity to apply appropriate actions to solve the problem, rather than repetitively thinking about negative consequences (Garnefski et al., 2001). The more repetitive thinking about misinterpretations, the less application of practical plans. On the other hand, the relationship between interpretation bias and suppression was not significant. It might be because suppression is not necessarily a maladaptive strategy regarding its context (Bardeen and Fergus, 2014). Therefore, individuals with higher and lower levels of interpretation bias might apply suppression equally; making this strategy independent of interpretation bias. This finding is consistent with those of Gillian et al., (2010), who argued high catastrophizers did not necessarily apply suppression to regulate their emotions.

This study had some limitations that should be considered when interpreting the findings. The samples in the IAD and control groups were university students; it restricts generalizing the results to other populations. Besides, the research samples were recruited through a volunteer catchment rather than those seeking help in clinics. Therefore, the level of dysfunctionality in our sample might be less than those who felt the need of visiting a psychologist. This may increase the odds of typeII error in our analyses, which has to be addressed when interpreting the results.

Despite the mentioned limitations, the present research was one of the few studies that compared ER strategies between IAD and control groups. Furthermore, it went beyond evaluating two main strategies of reappraisals and suppression. Based on our results, the less application of reappraisal and refocus on planning, as well as more usage of rumination and catastrophizing, could be crucial maintenance factors to prone individuals to more severe IAD cases. We also found how considering interpretation bias for health-related data may highlight the impact of ER in IAD. Interpretation bias could make individuals ruminatively think about the catastrophic consequences of bodily changes that interrupt more positive reappraisal or practical problem-solving strategies. This might increase the odds of experiencing more severe IAD types. However, further studies are required to explore the relationship between ER strategies and other components of IAD, like avoidance and sensitivity to bodily symptoms.

\section{Conclusion}

Both functional (e.g. reappraisal \& planning) and dysfunctional strategies (e.g. rumination \& catastrophizing) contributed to the psychopathology of IAD. The biased interpretation of bodily information could make individuals prone to ruminate about the catastrophic consequences of bodily changes; such conditions interrupt 
fostering more positive reappraisal or practical problemsolving strategies.

\section{Ethical Considerations}

\section{Compliance with ethical guidelines}

All ethical principles were considered in this article. The participants were informed about the purpose of the research and its implementation stages; they were also assured about the confidentiality of their information; Moreover, They were allowed to leave the study whenever they wish, and if desired, the results of the research would be available to them.

\section{Funding}

This research did not receive any specific grant from funding agencies in the public, commercial, or not-forprofit sectors.

\section{Authors' contributions}

All authors contributed equally in preparing all parts of the research.

\section{Conflict of interest}

The authors declared no conflicts of interest.

Acknowledgments

We would like to express our thanks to all participants who attended our study.

\section{References}

Abramowitz, J. S., Brigidi, B. D., \& Foa, E. B. (1999). Health concerns in patients with obsessive-compulsive disorder. Journal of Anxiety Disorders, 13(5), 529-39. [DOI:10.1016/S08876185(99)00022-5]

Akbari, F., Dehghani, M., Khatibi, A., \& Vervoort, T. (2016). Incorporating family function into chronic pain disability: the role of catastrophizing. Pain Research and Management, 2016, 6838596. [DOI:10.1155/2016/6838596] [PMID] [PMCID]

American Psychiatric Association. (2013). Diagnostic and Statistical Manual of mental disorders (DSM-5®). New York: American Psychiatric Association Publishing. [DOI:10.1176/appi books.9780890425596
Bardeen, J. R., \& Fergus, T. A. (2014). An examination of the incremental contribution of emotion regulation difficulties to health anxiety beyond specific emotion regulation strategies. Journal of Anxiety Disorders, 28(4), 394-401. [DOI:10.1016/j. janxdis.2014.03.002] [PMID]

Barsky, A. J., Ettner, S. L., Horsky, J., \& Bates, D. W. (2001) Resource utilization of patients with hypochondriacal health anxiety and somatization. Medical Care, 39(7), 705-15 [DOI:10.1097/00005650-200107000-00007] [PMID]

Campbell-Sills, L., Ellard, K., \& Barlow, D. (2013). Emotion regulation in anxiety disorders. In J. Gross, Handbook of Emotion Regulation. New York: Guilford Press. [DOI:10.1016/j. janxdis.2013.06.007] [PMID]

Cioffi, D., \& Holloway, J. (1993). Delayed costs of suppressed pain. Journal of Personality and Social Psychology, 64(2), 274-82. [DOI:10.1037/0022-3514.64.2.274] [PMID]

Deacon, B., \& Abramowitz, J. S. (2008). Is hypochondriasis related to obsessive-compulsive disorder, panic disorder, or both? An empirical evaluation. Journal of Cognitive Psychotherapy, 22(2), 115. [DOI:10.1891/0889-8391.22.2.115]

Fergus, T. A., \& Valentiner, D. P. (2010). Disease phobia and disease conviction are separate dimensions underlying hypochondriasis. Journal of Behavior Therapy and Experimental Psychiatry, 41(4), 438-44. [DOI:10.1016/j.jbtep.2010.05.002] [PMID]

First, M. B., Williams, J. B. W., Karg, R. S., \& Spitzer, R. L. (2015). Structured clinical interview for DSM-5 disorders, clinician version (SCID-5-CV). Arlington, VA: American Psychiatric Association. https:// books.google.com/books?id=TqftrQEACAAJ\&dq

Garnefski, N., Kraaij, V., \& Spinhoven, P. (2001). Negative life events, cognitive emotion regulation and emotional problems. Personality and Individual Differences, 30(8), 1311-27. [DOI:10.1016/S0191-8869(00)00113-6]

Gerolimatos, L. A., \& Edelstein, B. A. (2012). Predictors of health anxiety among older and young adults. International Psychogeriatrics, 24(12), 1998-2008. [DOI:10.1017/S1041610212001329] [PMID]

Gilliam, W., Burns, J. W., Quartana, P., Matsuura, J., Nappi, C., \& Wolff, B. (2010). Interactive effects of catastrophizing and suppression on responses to acute pain: A test of an appraisal $\times$ emotion regulation model. Journal of Behavioral Medicine 33(3), 191-9. [DOI:10.1007/s10865-009-9245-0] [PMID] [PMCID]

Görgen, S. M., Hiller, W., \& Witthöft, M. (2014). Health anxiety, cognitive coping, and emotion regulation: A latent variable approach. International Journal of Behavioral Medicine, 21(2), 364-74. [DOI:10.1007/s12529-013-9297-y] [PMID]

Gross, J. J. (1998). The emerging field of emotion regulation: An integrative review. Review of General Psychology, 2(3), 271 [DOI:10.1037/1089-2680.2.3.271]

Gross, J. J., \& John, O. P. (2003). Individual differences in two emotion regulation processes: Implications for affect, relationships, and well-being. Journal of Personality and Social Psychology, 85(2), 348. [DOI:10.1037/0022-3514.85.2.348] [PMID]

Gross, J. J., \& Levenson, R. W. (1993). Emotional suppression: Physiology, self-report, and expressive behavior. Journal of Personality and Social Psychology, 64(6), 970-86. [DOI:10.1037/00223514.64.6.970] [PMID] 
Haenen, M. A., de Jong, P. J., Schmidt, A. J., Stevens, S., \& Visser, L. (2000). Hypochondriacs' estimation of negative outcomes: domain-specificity and responsiveness to reassuring and alarming information. Behaviour Research and Therapy, 38(8), 819-33. [DOI:10.1016/S0005-7967(99)00128-X]

Jacob, S., \& Anto, M. M. (2016). A study on cognitive emotion regulation and anxiety and depression in adults. The International Journal of Indian Psychology, 3(2), 118-24. http:/ / oaji.net/ articles/2016/1170-1452464986.pdf

Koster, E. H., Rassin, E., Crombez, G., \& Näring, G. W. (2003) The paradoxical effects of suppressing anxious thoughts during imminent threat. Behaviour Research and Therapy, 41(9), 1113-20. [DOI:10.1016/S0005-7967(03)00144-X]

Kulpa, M., Ziętalewicz, U., Kosowicz, M., Stypuła-Ciuba, B., \& Ziółkowska, P. (2016). Anxiety and depression and cognitive coping strategies and health locus of control in patients with ovary and uterus cancer during anticancer therapy. Contemporary Oncology, 20(2), 171-5. [DOI:10.5114/wo.2016.60074] [PMID] [PMCID]

Lyubomirsky, S., Tucker, K. L., Caldwell, N. D., \& Berg, K. (1999). Why ruminators are poor problem solvers: Clues from the phenomenology of dysphoric rumination. Journal of Personality and Social Psychology, 77(5), 1041-60. [DOI:10.1037/00223514.77.5.1041] [PMID]

Marcus, D. K., Gurley, J. R., Marchi, M. M., \& Bauer, C. (2007). Cognitive and perceptual variables in hypochondriasis and health anxiety: A systematic review. Clinical Psychology Review, 27(2), 127-39. [DOI:10.1016/j.cpr.2006.09.003] [PMID]

Marcus, D. K., Hughes, K. T., \& Arnau, R. C. (2008). Health anxiety, rumination, and negative affect: A mediational analysis. Journal of Psychosomatic Research, 64(5), 495-501. [DOI:10.1016/j. jpsychores.2008.02.004] [PMID]

Pilowsky, I. (1967). Dimensions of hypochondriasis. The British Journal of Psychiatry, 113(494), 89-93. [DOI:10.1192/ bjp.113.494.89] [PMID]

Rief, W., Hiller, W., \& Margraf, J. (1998). Cognitive aspects of hypochondriasis and the somatization syndrome. Journal of Abnormal Psychology, 107(4), 587-95. [DOI:10.1037/0021843X.107.4.587] [PMID]

Salkovskis, P. M., Rimes, K. A., Warwick, H. M. C., \& Clark, D. M. (2002). The Health Anxiety Inventory: Development and validation of scales for the measurement of health anxiety and hypochondriasis. Psychological Medicine, 32(5), 843-53. [DOI:10.1017/S0033291702005822] [PMID]

Warwick, H. M., \&Salkovskis, P. M. (1990). Hypochondriasis. Behaviour Research and Therapy, 28(2), 105-17. [DOI:10.1016/00057967(90)90023-C]

Weck, F., Neng, J. M., Richtberg, S., \& Stangier, U. (2012). Dysfunctional beliefs about symptoms and illness in patients with hypochondriasis. Psychosomatics, 53(2), 148-54. [DOI:10.1016/j. psym.2011.11.007] [PMID]

Wegner, D. M., Schneider, D. J., Carter, S. R., \& White, T. L. (1987). Paradoxical effects of thought suppression. Journal of Personality and Social Psychology, 53(1), 5. [DOI:10.1037/00223514.53.1.5] 